\title{
A Retrospective Study of Congenital Cardiac Abnormality Associated with Scoliosis
}

\author{
Evin Bozcali ${ }^{1}$, Hanifi Ucpunar ${ }^{2}$, Ahmet Sevencan $^{2}$, Mehmet Bulent Balioglu $^{2}$, Akif Albayrak ${ }^{2}$, Veli Polat ${ }^{3}$ \\ ${ }^{1}$ Department of Cardiology, Metin Sabanci Baltalimani Bone Diseases Training and Research Hospital, Istanbul, Turkey \\ ${ }^{2}$ Department of Orthopedics and Traumatology, Metin Sabanci Baltalimani Bone Diseases Training and Research Hospital, Istanbul, Turkey \\ ${ }^{3}$ Department of Cardiology, Bakirkoy Dr. Sadi Konuk Training and Research Hospital, Istanbul, Turkey
}

\begin{abstract}
Study Design: Retrospective study.
Purpose: To identify the incidence of congenital cardiac abnormalities in patients who had scoliosis and underwent surgical treatment for scoliosis.

Overview of Literature: Congenital and idiopathic scoliosis (IS) are associated with cardiac abnormalities. We sought to establish and compare the incidence of congenital cardiac abnormalities in patients with idiopathic and congenital scoliosis (CS) who underwent surgical treatment for scoliosis.

Methods: Ninety consecutive scoliosis patients, who underwent surgical correction of scoliosis, were classified as CS (55 patients, 28 female [51\%]) and IS (35 patients, 21 female [60\%]). The complete data of the patients, including medical records, plain radiograph and transthoracic echocardiography were retrospectively assessed.

Results: We found that mitral valve prolapse was the most common cardiac abnormality in both patients with IS (nine patients, 26\%) and CS (13 patients, 24\%). Other congenital cardiac abnormalities were atrial septal aneurysm (23\% of IS patients, $18 \%$ of CS patients), pulmonary insufficiency (20\% of IS patients, $4 \%$ of CS patients), aortic insufficiency (17\% of IS patients), atrial septal defect (11\% of IS patients, $13 \%$ of CS patients), patent foramen ovale (15\% of CS patients), dextrocardia (4\% of CS patients), bicuspid aortic valve (3\% of IS patients), aortic stenosis ( $2 \%$ of CS patients), ventricular septal defect (2\% of CS patients), and cardiomyopathy ( $2 \%$ of CS patients).

Conclusions: We determined the increased incidence of congenital cardiac abnormalities among patients with congenital and IS. Mitral valve prolapse appeared to be the most prevalent congenital cardiac abnormality in both groups.
\end{abstract}

Keywords: Congenital scoliosis; Idiopathic scoliosis; Echocardiography; Congenital heart abnormality

\section{Introduction}

Scoliosis, which is defined as a lateral curve of the spine of more than 10 degrees, is one of the most common spinal deformities. The most frequent type of scoliosis is idiopathic scoliosis (IS) that consists approximately $80 \%$ of the patients with scoliosis. The remaining $20 \%$ are the cases of congenital scoliosis (CS), neuromuscular scoliosis and scoliosis related to syndromes [1,2]. The cause of the IS is unknown and its diagnosis is made through excluding the causes for other types of scoliosis $[2,3]$. The CS is characterized by the failure of vertebral formation or

Received Jul 12, 2015; Revised Jul 24, 2015; Accepted Aug 01, 2015

Corresponding author: Evin Bozcali

Department of Cardiology, Koç Universitesi Hastanesi, Davutpaşa Caddesi No: 4, 34010, Topkapı, Istanbul, Turkey

Tel: +90-850-2508250-29484, Fax: +90-212-338-1168, E-mail: ebozcali@gmail.com 
segmentation during the embryonic development. The CS is frequently associated with other abnormalities of the spinal cord, genitourinary system, bowel, auditory, and the heart [1-4].

The relationship between spine deformities and major cardiac abnormalities is well known [5]. The frequency of scoliosis in patients with congenital heart disease was reported as $11 \%$ to $34 \%$. Previously, it was suggested that cyanosis associated with congenital heart anomalies is a potential risk factor for the development of scoliosis $[6,7]$. Thoracotomy for congenital heart disease or other conditions has been demonstrated for an elevated risk of scoliosis [8,9]. In addition, it has been shown that the incidence of scoliosis after sternotomy in comparison with thoracotomy was similar. The development of scoliosis was mostly linked to the existing cardiac condition rather than the surgical procedure. Also, IS was found 10 times more often in children with congenital heart disease [10].

The incidence of congenital heart defects in patients with CS has been reported as 30\% [1]. Several reports revealed an increase in the incidence of congenital heart abnormalities, namely mitral valve prolapse (MVP), atrial septal defect (ASD), ventricular septal defect (VSD), tricuspid regurgitation and mitral valve dysplasia, among the adolescent IS patients [11-13]. In the current study, we aimed to determine and compare the incidence of congenital cardiac abnormalities in patients with idiopathic and CS who underwent surgical treatment for scoliosis.

\section{Materials and Methods}

\section{Study design and subjects}

A total of 90 consecutive patients (mean age, $14 \pm 7 ; 49$ female) with scoliosis who underwent surgical correction for scoliosis at Metin Sabanci Baltalimani Bone Diseases Training and Research hospital in September 2010 and January 2014 were included in the study. Depending on the presence or absence of vertebral spinal anomalies, all patients with scoliosis were classified as CS (55 patients, 28 female [51\%]) or IS (35 patients, 21 female [60\%]). We excluded patients who had neuromuscular and syndromic scoliosis. Patients with the IS and CS underwent echocardiographic examination either for detecting the heart murmur or for monitoring other cardiac abnormalities. Cardiologists and pediatric cardiologists performed the preoperative echocardiographic examinations. The com- plete data of the patients, including medical records, plain radiographs, and transthoracic echocardiography were retrospectively evaluated.

\section{Statistical analysis}

Descriptive statistics were expressed as mean \pm standard deviation for continuous variables and percentage (\%) or frequency for categorical variables. Normal distribution of the variables was examined with the KolmogorovSmirnov test. Continuous variables were compared using the independent samples t-test. Categorical variables were compared with the chi-square test or Fisher exact test. A $p$-value $<0.05$ was considered statistically significant. All statistical analyses were performed using the software SPSS ver. 22.0 (IBM Co., Armonk, NY, USA).

\section{Results}

The mean age was significantly higher in patients with the IS than patients with the CS (mean age, $19 \pm 5$ vs. $11 \pm 7$ years, $p<0.001)$. Preoperative echocardiogram recordings revealed congenital cardiac abnormalities in 23 out of 35 patients (66\%) with the IS and 30 out of 55 patients (55\%) with the CS. Moreover, idiopathic and CS groups were comparable in respect to abnormal echocardiograms as shown in Table 1. In the IS group, incidences of the MVP, atrial septal aneurysm (ASA), pulmonary insufficiency, aortic insufficiency, bicuspid aortic valve (BAV), and ASD were $26 \%, 23 \%, 20 \%, 17 \%, 3 \%$, and $11 \%$, respectively. In the CS group, incidences of the MVP, ASA, patent foramen ovale (PFO), ASD, pulmonary insufficiency, dextrocardia, aortic stenosis (AS), cardiomyopathy, VSD were $24 \%, 18 \%, 15 \%, 13 \%, 4 \%, 4 \%, 2 \%, 2 \%$, and $2 \%$, respectively.

Both groups were similar for gender, rates of the MVP, ASA, BAV, AS, ASD, cardiomyopathy, VSD, and dextrocardia (Table 1). MVP was the most common cardiac abnormality in both patients with the IS (nine patients, 26\%) and the CS (13 patients, 24\%). Pulmonary insufficiency and aortic insufficiency were significantly more frequent in the IS compared to CS group (20\% of IS patients vs. $4 \%$ of CS patients, $p=0.01 ; 17 \%$ of IS patients vs. $0 \%$ of CS patients, $p=0.001$; respectively). Conversely, PFO was significantly more frequent in the CS group than in the IS ( $15 \%$ of CS patients vs. $0 \%$ of IS patients, $p=0.02$ ). The rates of other congenital cardiac abnormalities in patients 
Table 1. Demographic data and cardiac abnormalities of patients with idiopathic and congenital scoliosis

\begin{tabular}{|c|c|c|c|}
\hline Characteristics & $\begin{array}{l}\text { Idiopathic scoliosis } \\
\qquad(\mathrm{n}=35)\end{array}$ & $\begin{array}{l}\text { Congenital scoliosis } \\
\qquad(n=55)\end{array}$ & $p$-value \\
\hline Age (yr) & $19 \pm 5$ & $11 \pm 7$ & $<0.001$ \\
\hline Female, sex & $21(60)$ & $28(51)$ & NS \\
\hline Normal echocardiogram & $12(34)$ & $25(45)$ & NS \\
\hline Abnormal echocardiogram & $23(66)$ & $30(55)$ & NS \\
\hline MVP & $9(26)$ & $13(24)$ & NS \\
\hline ASA & $8(23)$ & $10(18)$ & NS \\
\hline $\mathrm{Pl}$ & $7(20)$ & $2(4)$ & 0.01 \\
\hline Al & $6(17)$ & 0 & 0.001 \\
\hline BAV & $1(3)$ & 0 & NS \\
\hline AS & 0 & $1(2)$ & NS \\
\hline ASD & $4(11)$ & $7(13)$ & NS \\
\hline Cardiomyopathy & 0 & $1(2)$ & NS \\
\hline PFO & 0 & $8(15)$ & 0.02 \\
\hline VSD & 0 & $1(2)$ & NS \\
\hline Dextrocardia & 0 & $2(4)$ & NS \\
\hline
\end{tabular}

Values are presented as mean \pm standard deviation or number $(\%)$.

NS, non-significant; MVP, mitral valve prolapse; ASA, atrial septal aneurysm; PI, pulmonary insufficiency; Al, aortic insufficiency; BAV, bicuspid aortic valve; AS, aortic stenosis; ASD, atrial septal defect; PFO, patent foramen ovale; VSD, ventricular septal defect.

with the IS and the CS are shown in Table 1. There were no patients who were required cardiac intervention prior to scoliosis surgery. Sixteen patients with congenital cardiac abnormality had infective endocarditis prophylaxis and one patient with cardiomyopathy had heart failure therapy according to current guidelines. No deterioration in any patient with congenital cardiac abnormality was observed after the scoliosis surgery.

\section{Discussion}

To the best of our knowledge, this is the first study to compare the prevalence of congenital cardiac abnormalities among patients with idiopathic versus CS. We retrospectively evaluated the preoperative echocardiographic examinations of scoliosis patients who had undergone surgical correction.

Sixty-one percent of the CS patients also had an additional congenital anomaly in other organ systems including cardiovascular system. Atrial or VSDs, Tetralogy of Fallot, and PFO was present in nearly $30 \%$ of CS patients. Routine echocardiogram screening is strongly recommended in all patients with the CS, especially before sur- gical treatment $[1,2]$. The incidence of congenital heart disease has been reported as $26 \%$ in one series of 126 patients with congenital spinal deformity [14]. The most frequent type of scoliosis is idiopathic, which is responsible for $80 \%$ of scoliosis in children and its etiology is unknown [2]. Although, IS is not associated with known comorbid disorders, Liu et al. [13] reported an increased incidence of cardiac abnormalities (25 of 80 patients, $31.25 \%$ ) in Asian patients with adolescent IS. Additionally, they demonstrated that the ASD was the most common structural cardiac abnormality ( 7 of 80 patients, $8.75 \%$ ) in the adolescent IS patients based on the echocardiographic examinations $[1,13]$. According to our findings, the prevalence of congenital cardiac abnormalities was 66\% (23 of 35 patients) in patients with IS and 55\% (30 of 55 patients) in patients with CS. Both groups were statistically similar in respect to the prevalence of congenital cardiac abnormalities. Remarkably, our results indicated more frequent cardiac abnormalities in patients with congenital and IS compared to the cases that have previously been reported. The higher prevalence of cardiac abnormalities can be explained through: (1) the fact that the IS group includes a small number of patients, and therefore the true 
incidence might not have been reflected in these patients; (2) the fact that patients who had heart murmur or doubt of possible heart disease, underwent echocardiographic examination; and (3) the fact that both cardiac and skeletal systems could be influenced by a connective tissue disorder, and therefore the aortic and pulmonary insufficiencies, which are valvular heart disease and they may be related to connective tissue disorder, were significantly more frequent in the IS patients than the CS patients in our findings.

Increased prevalence of the MVP among patients with IS has been shown in several previous studies [11-16]. Recently, the MVP was also shown to be the most frequent cardiac abnormality in the CS patients; in addition ASD, VSD, BAV, patent ductus arteriosus have been shown as other congenital heart diseases in the CS patients [17]. Hirschfeld et al. [11] and Primiano et al. [15] reported the rate of the MVP as $28 \%$ and $25 \%$ in patients with IS, respectively. Additionally, Dhuper et al. [12] demonstrated significantly higher incidence of the MVP among the IS patients compared to controls (13.6\% vs. $3.2 \%)$. Colomina et al. [16] recently reported a $24.4 \%$ prevalence of cardiac valvular anomalies in patients with IS and they found the MVP as a most common cardiac valvular disorder (17 of 23 patients) in these patients.

In line with these previous findings, our results revealed that the MVP was the most frequent congenital cardiac abnormality in both IS and CS patients, which was detected $26 \%$ of patients with IS and $24 \%$ of patients with CS. An association between the MVP and thoracic skeletal abnormalities has been reported and a connective tissue disorder that simultaneously affects the embryonic development of both thoracic cage and atrioventricular valves has been suggested as an underlying cause of both conditions $[18,19]$. Although the relationship between the MVP and the IS has not been established, it has been supposed that development of geometrical changes of the mitral ring and left ventricle due to constriction of forward bending of the thoracic spine to the heart may produce relative elongation of the mitral valve chorda tendinea and eventually the formation of the MVP [20]. However, the MVP was still present following the surgical treatment of spinal deformity, indicating that geometric changes in the heart may not cause the development of the MVP in patients with IS. Genetic factors can also be responsible for both the development of MVP and IS [12].

In the present study, we demonstrated that ASA was the second most common congenital cardiac abnormality in patients with IS and CS. To our knowledge, the frequency of the ASA in these patients has not been previously reported. The incidence of the ASA was $23 \%$ in the IS patients and $18 \%$ in the CS patients, and the incidence was statistically similar in both groups. The ASA can develop secondary to interatrial pressure variations or might be a congenital malformation and it may also be associated with other cardiac abnormalities, including ASD, PFO, and MVP [21]. We suggest that coexistence of the ASA and scoliosis might be explained by an underlying connective tissue disorder.

Aortic and pulmonary insufficiencies, which are functional cardiac abnormalities, were significantly more frequent in patients with IS than in those with CS. Otherwise, $\mathrm{PFO}$, which is a structural cardiac abnormality, was significantly more common in the CS patients than in the IS patients. We may postulate that functional cardiac abnormalities are more frequently associated with the IS, while structural cardiac abnormalities are more common in patients with CS.

Hereby, we strongly recommend that routine echocardiography be performed in every patient with idiopathic and CS before the surgical treatment for scoliosis. Furthermore, echocardiographic examination should not be limited to scoliosis patients undergoing surgical correction. We also highly recommend echocardiographic evaluation in all scoliosis patients both idiopathic and congenital.

There were several limitations to the present study that need to be addressed. A major limitation was the relatively small number of patients in each group of the study, which may not be large enough to manifest a more accurate incidence of the congenital heart disease in patients with idiopathic and CS. Another limitation was the retrospective nature of this study. Thereby, preoperative echocardiographic examination could not be performed in all patients who had undergone surgical correction of scoliosis. The last limitation of the study is that it was restricted to scoliosis patients who underwent surgical treatment instead of all patients with IS and CS.

\section{Conclusions}

Although congenital cardiac anomalies associated with scoliosis are mostly benign, it is still necessary to identify the associated congenital cardiac abnormality. Rarely, 
however, congenital cardiac abnormality can be serious and preoperative measures might be required to prevent poor outcome after surgery. In this regard, these patients can be aware of their congenital cardiac disease and they can be informed regarding the therapy and follow-up of the congenital cardiac abnormality.

\section{Conflict of Interest}

No potential conflict of interest relevant to this article was reported.

\section{References}

1. Horan MP, Milbrandt TA. Scoliosis in pediatric patients: comorbid disorders and screening. Ped Health 2006;3:451-6.

2. El-Hawary R, Chukwunyerenwa C. Update on evaluation and treatment of scoliosis. Pediatr Clin North Am 2014;61:1223-41.

3. Campos MA, Weinstein SL. Pediatric scoliosis and kyphosis. Neurosurg Clin N Am 2007;18:515-29.

4. Kose N, Campbell RM. Congenital scoliosis. Med Sci Monit 2004;10:RA104-10.

5. Ogilvie JW. Congenital heart disease and scoliosis. In: Lonstein JE, Winter RB, Bradford DS, Ogilvie JW, editors. Moe's textbook of scoliosis and other spinal deformities. Philadelphia, PA: W.B. Saunders Co; 1995. p.564-5.

6. Kawakami N, Mimatsu K, Deguchi M, Kato F, Maki S. Scoliosis and congenital heart disease. Spine (Phila Pa 1976) 1995;20:1252-5.

7. Roth A, Rosenthal A, Hall JE, Mizel M. Scoliosis and congenital heart disease. Clin Orthop Relat Res 1973;(93): 95-102.

8. Durning RP, Scoles PV, Fox OD. Scoliosis after thoracotomy in tracheoesophageal fistula patients. A follow-up study. J Bone Joint Surg Am 1980;62:11569.

9. Bisgard JD. Thoracogenic scoliosis influence of thoracic disease and thoracic operations on the spine. Arch Surg 1934;29:417-45.

10. Herrera-Soto JA, Vander Have KL, Barry-Lane P,
Myers JL. Retrospective study on the development of spinal deformities following sternotomy for congenital heart disease. Spine (Phila Pa 1976) 2007;32:19982004.

11. Hirschfeld SS, Rudner C, Nash CL Jr, Nussbaum E, Brower EM. Incidence of mitral valve prolapse in adolescent scoliosis and thoracic hypokyphosis. Pediatrics 1982;70:451-4.

12. Dhuper S, Ehlers KH, Fatica NS, et al. Incidence and risk factors for mitral valve prolapse in severe adolescent idiopathic scoliosis. Pediatr Cardiol 1997;18:425-8.

13. Liu L, Xiu P, Li Q, Song Y, Chen R, Zhou C. Prevalence of cardiac dysfunction and abnormalities in patients with adolescent idiopathic scoliosis requiring surgery. Orthopedics 2010;33:882.

14. Basu PS, Elsebaie H, Noordeen MH. Congenital spinal deformity: a comprehensive assessment at presentation. Spine (Phila Pa 1976) 2002;27:2255-9.

15. Primiano FP Jr, Nussbaum E, Hirschfeld SS, et al. Early echocardiographic and pulmonary function findings in idiopathic scoliosis. J Pediatr Orthop 1983;3:475-81.

16. Colomina MJ, Puig L, Godet C, Villanueva C, Bago J. Prevalence of asymptomatic cardiac valve anomalies in idiopathic scoliosis. Pediatr Cardiol 2002;23:426-9.

17. Liu YT, Guo LL, Tian Z, et al. A retrospective study of congenital scoliosis and associated cardiac and intraspinal abnormities in a Chinese population. Eur Spine J 2011;20:2111-4.

18. Salomon J, Shah PM, Heinle RA. Thoracic skeletal abnormalities in idiopathic mitral valve prolapse. Am J Cardiol 1975;36:32-6.

19. Udoshi MB, Shah A, Fisher VJ, Dolgin M. Incidence of mitral valve prolapse in subjects with thoracic skeletal abnormalities: a prospective study. Am Heart J 1979;97:303-11.

20. Yazawa Y. Mitral valve prolapse related to geometrical changes of the heart in cases of progressive muscular dystrophy. Clin Cardiol 1984;7:198-204.

21. Mugge A, Daniel WG, Angermann C, et al. Atrial septal aneurysm in adult patients. A multicenter study using transthoracic and transesophageal echocardiography. Circulation 1995;91:2785-92. 